\title{
Numerical Investigations on Aerodynamic Blockage Characteristics and Exit Blockage Prediction Model for Transonic Centrifugal Impellers
}

\author{
ZHANG Chaowei ${ }^{1,2}$, DONG Xuezhi ${ }^{1,2, *}$, LIU Xiyang ${ }^{1,2}$, GAO Qing $^{1,2}$, TAN Chunqing $^{1,2}$ \\ 1 Institute of Engineering Thermophysics, Chinese Academy of Sciences, Beijing 100190, P. R. China \\ 2 University of Chinese Academy of Sciences, Beijing 100049, P. R. China \\ E-mail: dongxuezhi@iet.cn
}

\begin{abstract}
This paper investigates the effect of the exit flow coefficient, rotational speed and tip clearance size on the exit aerodynamic blockage. An improved model is established to predict the exit aerodynamic blockage for transonic centrifugal impellers. The goal of numerical analysis and modeling is to supply an effective means of estimating exit aerodynamic blockage for use in the performance prediction stage.

The numerical results show that decreased exit flow coefficient, enhanced rotational speed, and increased tip clearance size can increase the exit aerodynamic blockage. The inlet blockage contributes to the exit blockage and it is mainly affected by the interaction of the shock wave with the tip leakage vortex. The improved model considers the interaction of the shock wave with the tip leakage vortex, based on the conventional model. Compared with the conventional model, the improved model shows better accuracy, with an average relative error below $9.79 \%$.
\end{abstract}

\section{INTRODUCTION}

In a centrifugal compressor design system, an effective performance prediction method is the backbone of the design process due to its guidance in preliminary design. In the process of performance prediction, most unknown parameters, such as exit aerodynamic blockage factor, slip factor, losses and so on, are dependent on empirical correlations or assumption (Casey and Robinson, 2010). Of these parameters, the exit aerodynamic blockage factor plays an important role in predicting the exit flow parameters and overall performance. It is necessary to investigate the exit aerodynamic blockage characteristics and proposed a suitable prediction model.

In the past few decades, aerodynamic blockage has been studied using the numerical and experimental approaches. By experiments and analysis, Senoo found that the exit aerodynamic blockage was correlated with diffusion ratio, passage friction and the effective blade camberline caused by the boundary layer accumulation for a centrifugal blower (Senoo et al., 1974). Pampreen found that the exit aerodynamic blockage was affected by the diffusion ratio, flowpath curvature, blade curvature and Coriolis acceleration for transonic centrifugal impellers by a series of experiments (Pampreen, 1981). Chima found that the tip leakage flow had a significant influence on blockage generation for transonic axial impellers by numerical simulations (Chima, 1998). Rodgers found the inlet blockage had a great effect on the exit blockage by examining the experimental data. (Rodgers, 1978). Ibaraki et al. found that the boundary layer flow on the suction surface was separated by the interaction with shock for a transonic centrifugal impeller by numerical simulations (Ibaraki et al., 2007). The blockage near the impeller inlet was mainly formed by tip leakage vortex (TLV), which enhanced its blockage effect by the interaction with shock wave. The numerical results indicated that the splitter blades could reduce the blockage effect (Kaneko and Tsujita, 2015). Yamada et al. reported that the intensity of the interaction of the shock wave with the tip leakage vortex relied on three factors by numerical simulations. One was the swirl ratio which represented the ratio of the TLV swirl velocity component to the TLV axial velocity component. Another was the static pressure difference between the upstream and downstream of the shock wave. The other was an angle formed by TLV and shock wave in the interaction. (Yamada et al., 2007). For a transonic centrifugal impeller, a significant reduction of the wake region at the impeller exit was found with vaned diffuser compared with the vaneless from a series of experimental results (Kai et al., 2002).

Some models have been proposed to predict the exit aerodynamic blockage factor. Before the empirical model presented, the exit aerodynamic blockage factor was assumed constant, even though the operation condition changed. Rodgers simplified the impeller channel as a twodimensional diffuser. Based on the assumption of a thin inlet 
boundary layer condition, the following model was proposed as Eq. (1) (Rodgers, 1978). However, this model implies that the exit aerodynamic blockage only depends on the geometrical structure, and is not affected by the flow parameters, when the inlet blockage is assumed constant.

$$
\begin{gathered}
B_{2}=0.07 A R+B_{1}^{0.67}-0.06 \\
A R=\frac{A_{2}}{A_{1 t h}}=\frac{2 \pi r_{2} b_{2}-Z_{2} b_{2} t_{2} / \cos \beta_{2 b}}{b_{1}\left(2 \pi r_{1, m} \cos \beta_{1 b, m}-Z_{1} t_{1}\right)}
\end{gathered}
$$

If the inlet boundary condition was characterized at the throat, the diffusion ratio in the uncovered section of the channel would be the critical dependence. Rodgers proposed a model to estimate the exit blockage, where the inlet blockage relied on the diffusion ratio (Rodgers, 1978).

$$
B_{2}=0.07 D R+\text { const } \cdot D R^{n}-0.06
$$

Diffusion ratio

$$
D R=\frac{\overline{W_{1}}}{\overline{W_{2}}}
$$

Oh proposed a blockage prediction model as Eq. (5), considering the effect of the tip clearance size, based on Rodgers' model (Oh and Oh, 2000).

$$
B_{2}=0.02 A R+0.03 D R^{3}+c / b_{2}
$$

However, these models don't consider the effect of the interaction of the shock wave with the tip leakage vortex on the inlet blockage, which has a great influence on the exit blockage.

In order to exclude the effect of the splitter blades and vaned diffuser on the exit blockage, centrifugal impellers followed by a vaneless diffuser are utilized in this study. This study investigates the effect of the exit flow coefficient, rotational speed and tip clearance size on the exit blockage. An improved model to predict the exit blockage for transonic centrifugal impellers is developed. The model considers the interaction of the shock wave with the tip leakage vortex, based on Oh's model, and it is validated with CFD.

\section{COMPUTATION METHOD}

Table 1 represents the summarized information of the centrifugal impeller called Krain4 impeller in this study. As shown in Figure 1, Krain4 impeller without splitter blades is followed by a vaneless diffuser. Krain4 impeller is open to the public with the three-dimensional geometry data, and the corresponding experimental performance data is available (Hah and Krain, 1990, Krain, 1984, Krain, 1988).

Table 1 Design parameters of Krain4 impeller

\begin{tabular}{cc}
\hline Design Parameter & Value \\
\hline Corrected mass flow $(\mathrm{kg} / \mathrm{s})$ & 4.0 \\
Stage pressure ratio & 4.1 \\
Corrected Rotational speed / rpm & 22363 \\
Impeller diameter $d_{2} / \mathrm{mm}$ & 400 \\
Exit blade angle $\beta_{2 \mathrm{~b}} / \mathrm{deg}$ & 30 \\
Blade width $b_{2} / \mathrm{mm}$ & 14.71 \\
Blade number & 24 \\
Tip clearance size $/ \mathrm{mm}$ & $0.5-0.3$ (LE-TE) \\
Inlet tip relative Mach Number & 0.92 \\
\hline
\end{tabular}

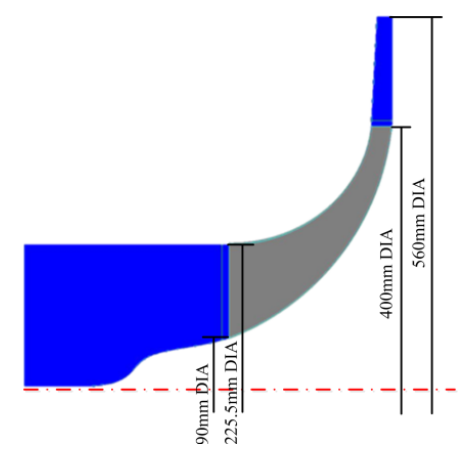

Figure 1 Geometry structure

Fine/Autogrid5 was used to generate the multi-block structured grids, which selected the default topology. The number of the grid was about 1.16 million. In addition, 17 nodes were used in the tip clearance region. To assure the computational accuracy, near-wall grid was refined and Yplus was less than 2 to meet the demand of solver. The grid number measured up the requirements of the computation model with the grid dependence test as shown in Figure 2.

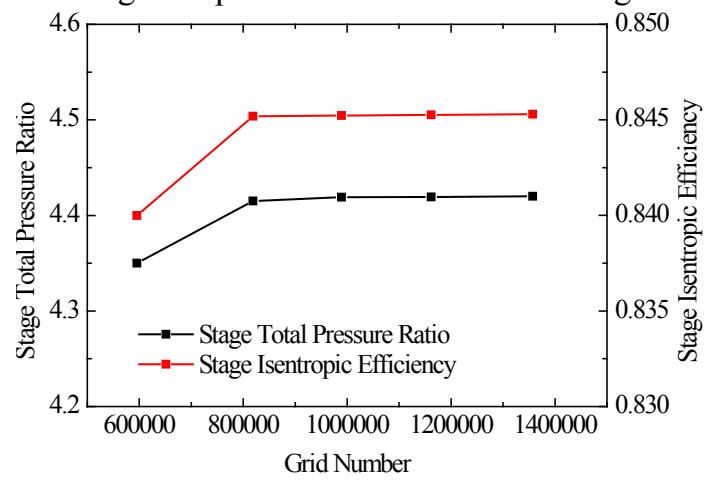

Figure 2 Grid independence study

S-A and SST turbulence models were compared against the available experimental data to select the more suitable turbulence model for our study. As shown in Figure 3, the performance curve presented by S-A model matched better with the experiment than that provided by SST model, whether no matter what the stall-onset point or pressure ratio is. In addition, according to the open literature, S-A model predicts the tip leakage flow of impellers well ( $\mathrm{Du}$ et al., 2013). Therefore, the S-A model was utilized in this study.

Under the different operating conditions, the flow was simulated by solving the steady Reynolds averaged N-S equation with S-A model in this study. Fine/Turbo was used to simulate the flows in the impeller. The total temperature and total pressure at inlet were $288.15 \mathrm{~K}$ and $101325 \mathrm{~Pa}$ respectively. The flow direction at inlet was axial. No-slip and adiabatic conditions were imposed on all solid walls. The steady simulations were carried out by the exit static pressure increased gradually until the last stable point was obtained. The simulation was thought to be converged when the following conditions were met: (1) the RMS residuals fallen below $1.0^{-5}$; (2) the difference of mass flow between inlet and outlet was less than $0.5 \%$; (3) the variation of efficiency was less than $0.01 \%$.

The predicted total pressure ratio is on average roughly $8.24 \%$ above the experimental value, and the choke mass 
flow rate is overestimated as shown in Figure 3. This deviation may be due to that the non-uniform distribution of the inlet flow parameters, but it is unknown in the experiment. The another reason may be the difference between CFD and experiments in air's physical parameters, such as humidity, which is unknown in the experiment. The other reason may be the throat area difference between simulation model and test model. Compared with the test model, the simulation model ignores the blade root fillet, which is known to decrease the total pressure ratio, the efficiency and the choke flow rate (Yi et al., 2014). These results indicate that the numerical scheme in this study predicts the overall performance reasonably well as a whole.

Figure 4 shows the comparison between the CFD predicted and measured circumferentially averaged static pressure distribution along the streamwise direction on the shroud under the different conditions for Impeller (C). The static pressure is normalized by the inlet total pressure. At the small and design mass flow point, the numerical predictions have great agreement with the experiment data. But the static pressure on the shroud predicted by CFD is a little greater than the experimental value near the choke $(m=4.6 \mathrm{~kg} / \mathrm{s})$. This might be due to the calculated mass flow rate being slightly higher than the measurement even though the exit static pressure is maintained the same as the measurement. Therefore, the computation methods in this study are validated for investigating the flow behaviour.

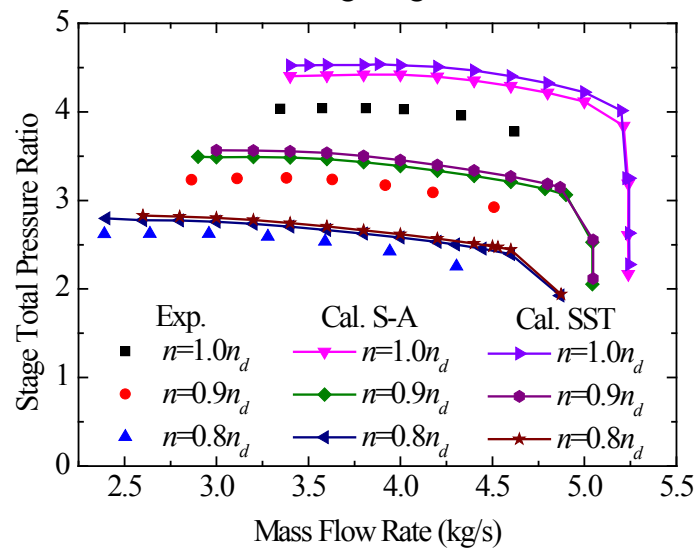

(a) Stage total pressure ratio

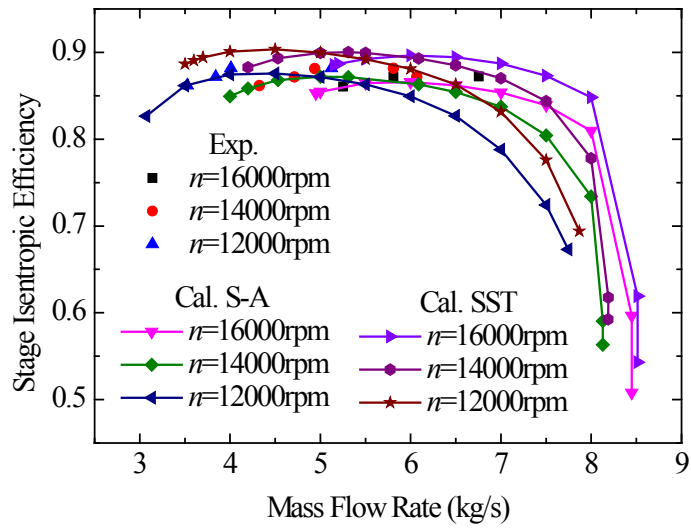

(b) Impeller polytropic efficiency

Figure 3 Comparison between the CFD predicted and measured performance

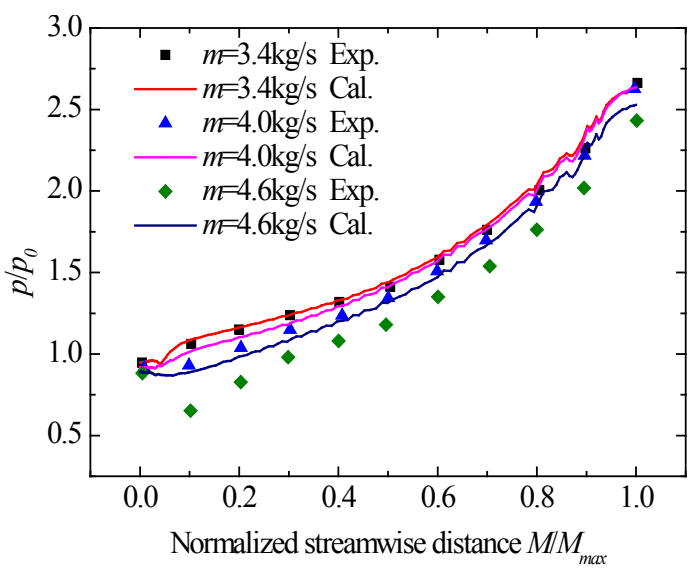

Figure 4 Comparison between the CFD predicted and measured static pressure on the shroud

\section{NUMERICAL EXIT AERODYNAMIC BLOCKAGE CHARACTERISTICS}

The aerodynamic blockage at the impeller exit is mainly caused by the boundary layer accumulation and secondary flow. As shown in Figure 5, the secondary flow structures consist of circumferential secondary flow, radial secondary flow and tip leakage flow. The circumferential secondary flow causes the low-energy fluid to accumulate on the suction surface. The radial secondary flow and tip leakage flow cause the low-energy fluid to accumulate on the shroud.

Circumferential Secondary Flow Tip Leakage Secondary Flow

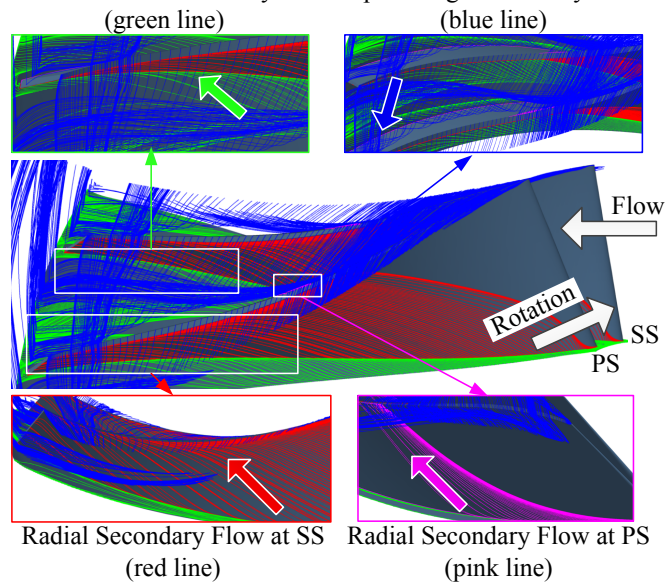

Figure 5 Secondary flow structures

The characteristics of the exit aerodynamic blockage are examined with exit flow coefficient, rotational speed, and tip clearance size. The exit aerodynamic blockage factor is used to evaluate the exit aerodynamic blockage. The numerical exit aerodynamic blockage factor is calculated using the mass-averaged data at the impeller exit plane as Eq. (6).

$$
\begin{gathered}
B_{2 N}=1-\frac{\overline{A_{2, e f f}}}{A_{2}} \\
A_{2}=2 \pi r_{2} b_{2}-\frac{b_{2} t_{2} Z_{2}}{\cos \beta_{2 b}} \\
\overline{A_{2, \text { eff }}}=\frac{m}{\overline{\rho_{2} V_{m 2}}}
\end{gathered}
$$




\section{Effect of exit flow coefficient}

The shock wave is formed by the flow acceleration along the suction surface. Near the stall condition, the lowenergy region at the downstream of the shock wave at $95 \%$ span as shown in Figure 6 (a). This is due to the fact that the tip leakage flow is diffused with the increase in diameter and attenuated by the interaction with the shock wave which has the strong adverse pressure gradient. With decreased exit flow coefficient, the shock wave moves upstream as shown in Figure 6 and its adverse pressure gradient becomes somewhat steeper as shown in Figure 7. Consequently, the inlet blockage effect caused by the adverse pressure gradient of the shock wave is increased. The decrease of exit flow coefficient increases the incidence angle and blade loading near the blade tip at the leading edge as shown in Figure 8. The decrease of the TLV streamwise momentum by the reduction of the exit flow coefficient and the decrease of the TLV swirl velocity component by the increase of the blade loading near the blade tip at the leading edge, both of them increase the swirl ratio. As a result, the inlet blockage effect caused by the swirl ratio is increased. The inlet blockage effect is enhanced by the rotational axis of TLV turned more in the circumferential direction as shown in Figure 6.

With decreased exit flow coefficient, the blade loading near the blade tip at the trailing edge increases as shown in Figure 8 , which intensifies the tip leakage flow and other secondary flows at the impeller exit. The enhancement of the inlet blockage and the intensified tip leakage flow and secondary flows at the impeller exit, increase the low-energy region near the shroud at the impeller exit, as shown in Figure 9. Therefore, the exit blockage increases, and the slope is high near the stall condition as shown in Figure 10.

The exit flow coefficient is defined according to the Eq. (9). The static pressure coefficient is defined as Eq. (10).

$$
\begin{gathered}
\phi_{2}=\frac{V_{2 m}}{U_{2}} \\
C_{p s}=\frac{p}{0.5 \rho_{01} U_{2}^{2}}
\end{gathered}
$$

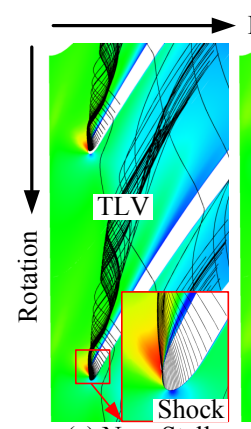

(a) Near Stall $\phi_{2}=0.227$

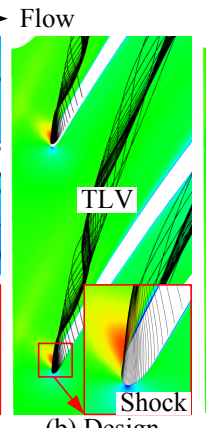

(b) Design $\phi_{2}=0.249$
Figure 6 Interaction of shock wave with tip leakage vortex with exit flow coefficient at $95 \%$ span

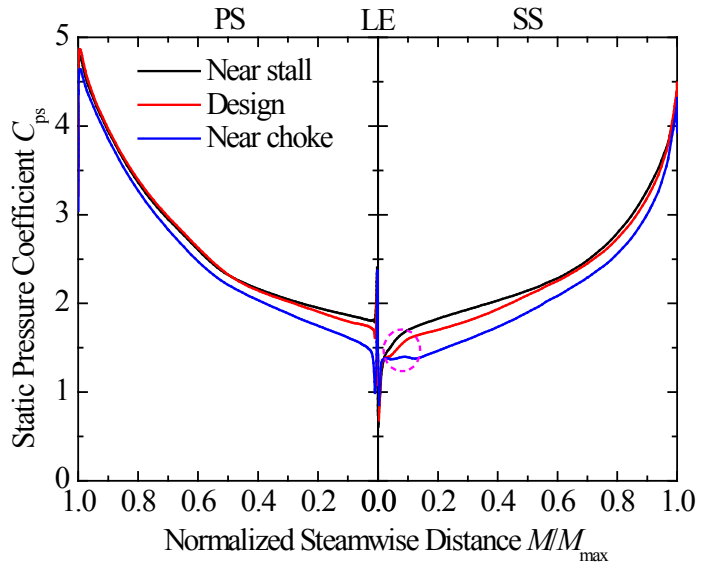

Figure 7 95\%span static pressure coefficient with exit flow coefficient

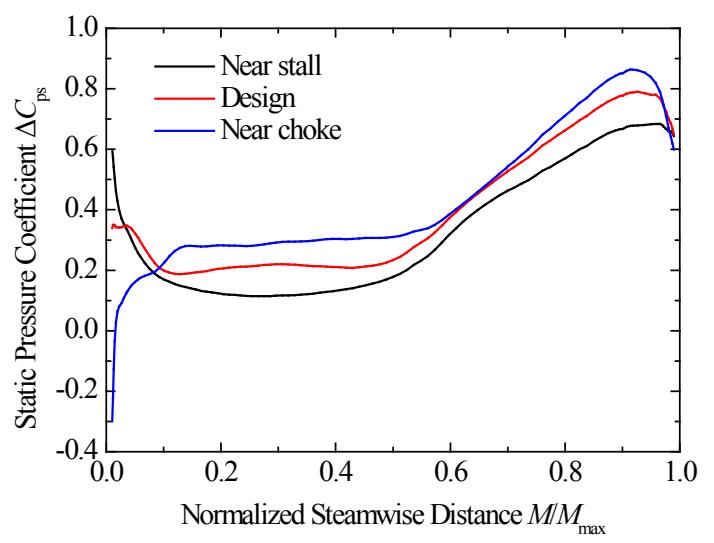

Figure 8 95\%span static pressure coefficient difference between the blade surfaces with exit flow coefficient

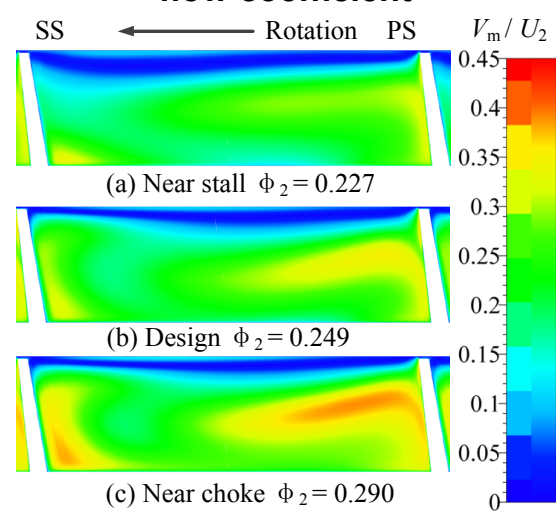

Figure 9 Meridional velocity at the impeller exit with exit flow coefficient 


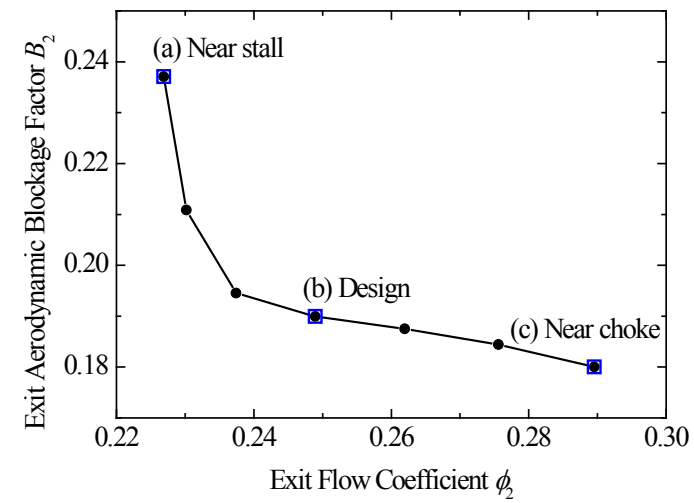

Figure 10 Numerical exit aerodynamic blockage factor with exit flow coefficient

\section{Effect of Rotational Speed}

When exit flow coefficient is kept unchanged, with decreased rotational speed, the intensity of the shock wave decreases and even disappears at $0.7 n_{\mathrm{d}}$. The inlet blockage effect caused by shock wave is decreased, especially a lot drop for exit blockage at $0.7 n_{\mathrm{d}}$ as shown in Figure 11. The decrease of rotational speed increases the incidence angle and the blade loading near the blade tip at the leading edge as shown in Figure 12. The inlet blockage effect is enhanced by the swirl ratio increased and the rotational axis of TLV turned more in the circumferential direction as shown in Figure 13. To sum up, the inlet blockage increases as the decrease of rotational speed.

As shown in Figure 12, with decreased rotational speed, the blade loading near the blade tip at the trailing edge decreases, which weaken the tip leakage flow and other secondary flows at the impeller exit. The tip leakage flow and other secondary flows at the impeller exit dominates the exit blockage, so the low-energy region increases near the shroud as shown in Figure 14, and the exit blockage increases as shown in Figure 11.

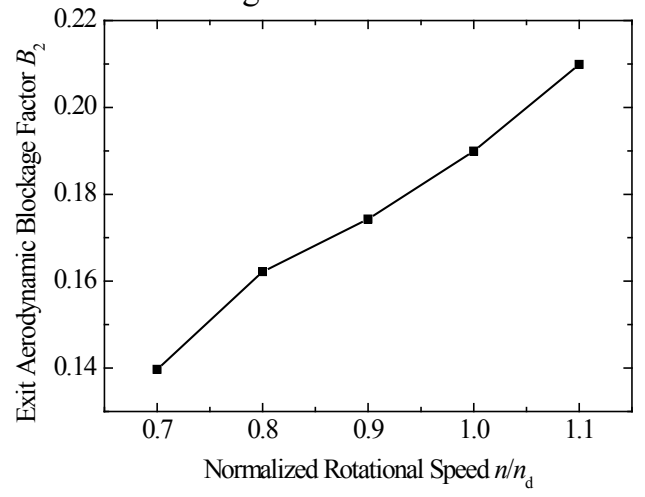

Figure 11 Numerical exit aerodynamic blockage factor with rotational speed $\left(\phi_{2}=0.249\right)$

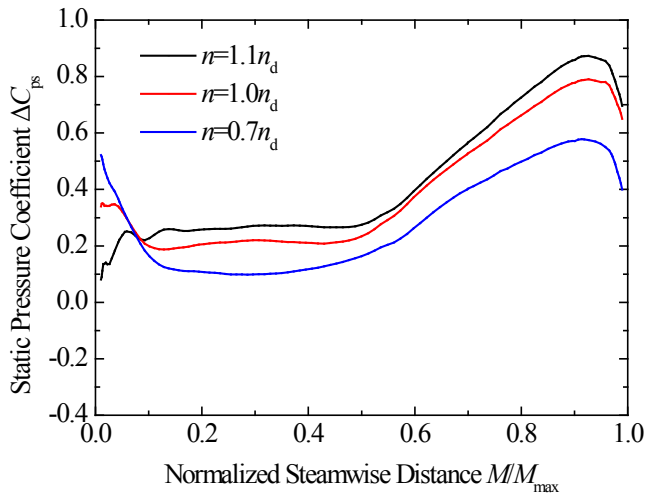

Figure 12 95\%span static pressure coefficient difference between the blade surfaces with rotational speed ( $\phi_{2}=0.249$ )

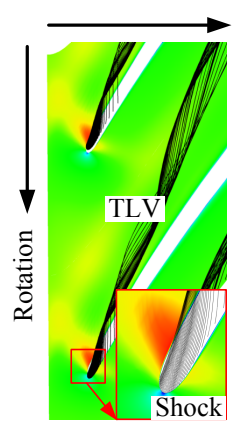

(a) $n / n_{\mathrm{d}}=1.1$

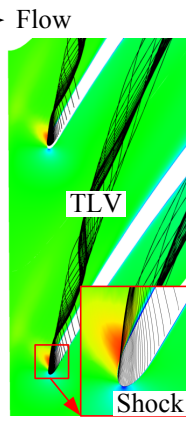

(b) $n / n_{\mathrm{d}}=1.0$

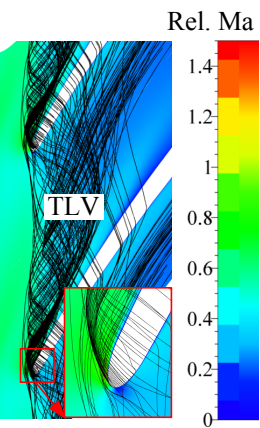

(c) $n / n_{\mathrm{d}}=0.7$
Figure 13 Interaction of shock wave with tip leakage vortex with rotational speed at $95 \%$ span $\left(\phi_{2}=\mathbf{0 . 2 4 9 )}\right.$

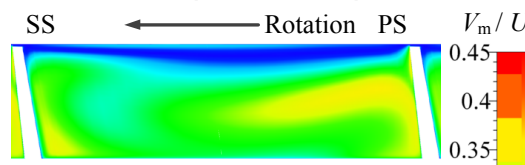

(a) $n / n_{\mathrm{d}}=1.1$

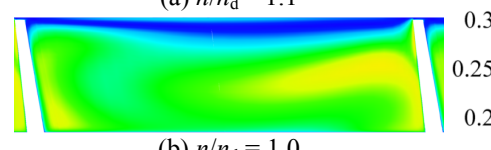

(b) $n / n_{\mathrm{d}}=1.0$

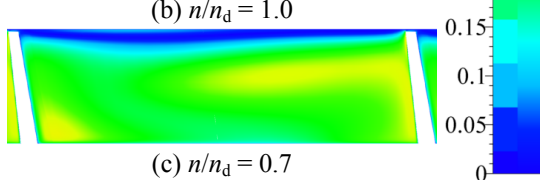

Figure 14 Meridional velocity at the impeller exit with rotational speed $\left(\phi_{2}=0.249\right)$

\section{Effect of Tip Clearance Size}

When exit flow coefficient is kept unchanged, with increased tip clearance size, the intensity of the shock wave is almost no changed as shown in Figure 15, which also can be expected from its adverse pressure gradient on the suction surface at the leading edge as shown in Figure 16. Therefore, the adverse pressure gradient of the shock wave has no effect on inlet blockage. The increase of tip clearance increases the blade loading near the blade tip at the leading edge as shown in Figure 17, which causes the swirl ratio to increase as shown in Figure 15. Consequently, the inlet blockage effect caused by the swirl ratio is increased. The inlet blockage 
effect is enhanced by the rotational axis of TLV turned more in the circumferential direction as shown in Figure 15. However, the inlet blockage almost has no change as shown in Figure 15. It indicates that the intensity of the shock wave dominates the inlet blockage, compared with tip clearance.

According to the increase of tip clearance size, the velocity of tip leakage flow at the trailing edge and the intensity of the circumferential and radial secondary flow decrease at the impeller exit by the reduction of the blade loading near the blade tip at the trailing edge, which decreases the exit blockage. However, the momentum of the tip leakage flow increases by the increased tip leakage flow rate, which contributes to the exit blockage. The low-energy region near the shroud at the impeller exit becomes large as shown in Figure 18. Therefore, with increased tip clearance size, the exit blockage increases, but the slope decreases gradually, as shown in Figure 19.

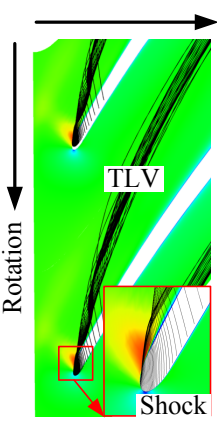

(a) $c / b_{2}=0.0136$

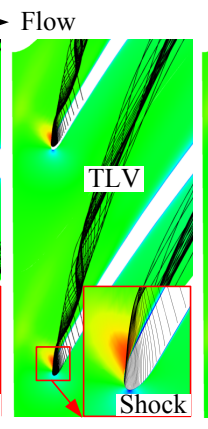

(b) $c / b_{2}=0.0272$

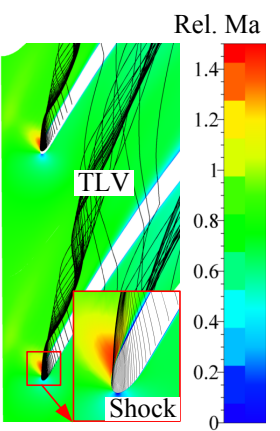

(c) $c / b_{2}=0.0408$
Figure 15 Interaction of shock wave with tip leakage vortex with tip clearance size at $95 \%$ span

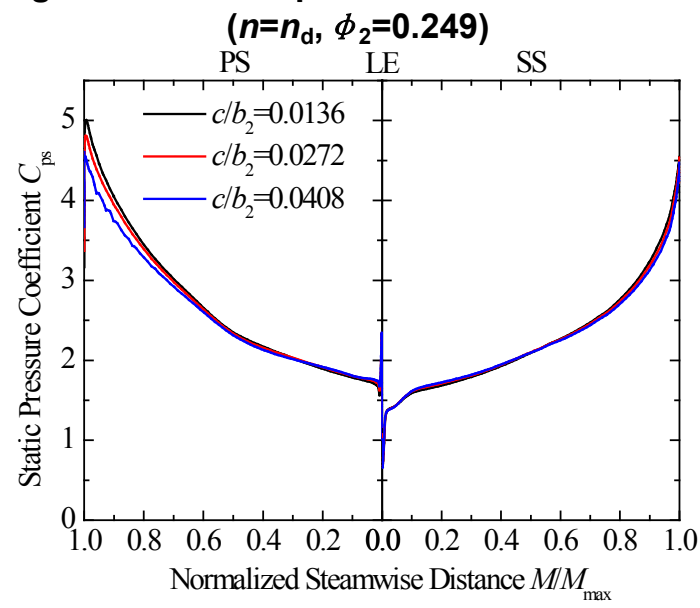

Figure $1695 \%$ span static pressure coefficient with tip clearance size $\left(n=n_{d}, \quad \phi_{2}=0.249\right)$

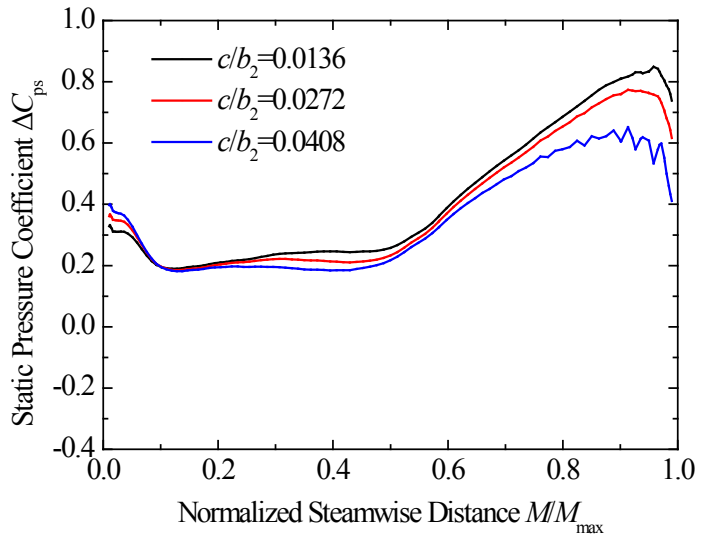

Figure 17 95\%span static pressure coefficient difference between the blade surfaces with tip clearance size $\left(n=n_{d}, \phi_{2}=0.249\right)$

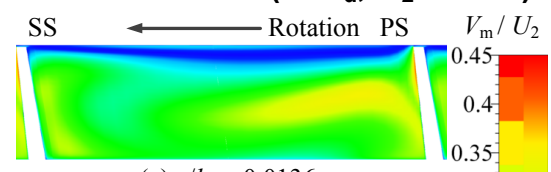

(a) $c / b_{2}=0.0136$

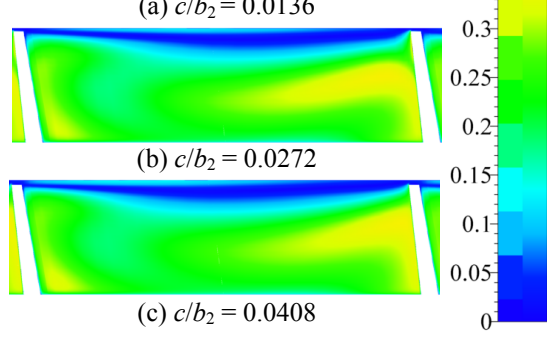

Figure 18 Meridional velocity at the impeller exit with tip clearance size $\left(n=n_{d}, \phi_{2}=0.249\right)$

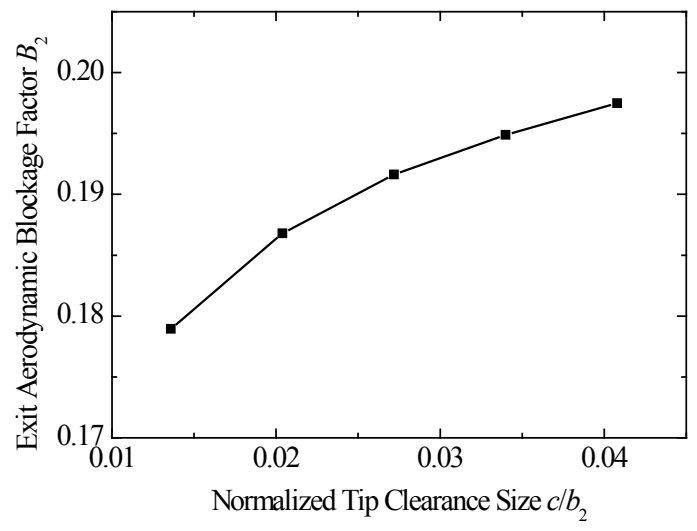

Figure 19 Numerical exit aerodynamic blockage factor with tip clearance size $\left(n=n_{d}, \quad \phi_{2}=0.249\right)$

\section{IMPROVED PREDICTION MODEL FOR EXIT AERODYNAMIC BLOCKAGE}

\section{Model Establishment}

According to the numerical results, the interaction of the shock wave with the tip leakage flow can increase the exit aerodynamic blockage. An improved model as Eq. (11) is proposed to predict exit blockage, considering the effect of shock wave, based on Oh's model. The inlet tip relative Mach number ratio defined as Eq. (13) is used to evaluate the intensity of shock wave. The constant coefficient $k$ is 
valued as 1.67 by fitting the numerical results of Krain4 impeller.

$$
\begin{gathered}
B_{2}=0.02 A R+0.03 D R^{3}+k \cdot C R \cdot M R \\
C R=\frac{c}{b_{2}} \\
M R=\left\{\begin{array}{cc}
\frac{M_{w 1 t}}{M_{w 1 t, c r}} & M_{w 1 t} \geq M_{w 1 t, c r} \\
1 & M_{w 1 t}<M_{w 1 t, c r}
\end{array}\right.
\end{gathered}
$$

where $M_{\mathrm{w} 1 \mathrm{t}, \mathrm{cr}}$ represents the critical inlet tip relative Mach number, valued as 0.8 .

\section{Model Validation}

To validate the improved model further, it is applied to another impeller, called Radiver impeller, without splitter blades and followed by a vaneless diffuser (Kai et al., 2002, Kai et al., 2003). This impeller is an open CFD test case, and its geometrical and experimental data as shown in Table 2 can be obtained on request at the Institute of Jet Propulsion and Turbomachinery of the RWTH Aachen. The computation method is same as that of Krain4 impeller. The grid test has been conducted and the computation method has been validated. Fine/Turbo was used to simulate the flows in the impeller under the different conditions.

Table 2 Design parameters of Radiver impeller

\begin{tabular}{cc}
\hline Design Parameter & Value \\
\hline Corrected mass flow $(\mathrm{kg} / \mathrm{s})$ & 2.5 \\
Stage pressure ratio & 4.07 \\
Corrected Rotational speed / rpm & 35200 \\
Impeller diameter $d_{2} / \mathrm{mm}$ & 270 \\
Exit blade angle $\beta_{2 \mathrm{~b}} / \mathrm{deg}$ & 38 \\
Blade width $b_{2} / \mathrm{mm}$ & 11.7 \\
Blade number & 15 \\
Tip clearance size $/ \mathrm{mm}$ & $0.7-0.48(\mathrm{LE}-\mathrm{TE})$ \\
Inlet tip relative Mach Number & 0.95 \\
Vaneless diffuser diameter $d_{3} / \mathrm{mm}$ & 670 \\
\hline
\end{tabular}

The relative error between the model predicted data and the numerical value is computed by Eq. (14). The RMSE of prediction model is expressed as Eq. (15).

$$
\begin{aligned}
& \text { Error }_{B_{2}, i}=\frac{\left|B_{2 M, i}-B_{2 N, i}\right|}{B_{2 N, i}} \times 100 \% \\
& \operatorname{RMSE}_{B_{2}}=\sqrt{\frac{1}{\text { num }} \sum_{i=1}^{i=n u m} \operatorname{Error}_{B_{2}, i}{ }^{2}}
\end{aligned}
$$

where subscript $i$ represents the $i$ th operation condition; num represents the amount of samples at the all operation conditions; Subscript $M$ represents the results predicted by model; Subscript $N$ represents the numerical results.

Figure 20 shows the exit aerodynamic blockage predicted by models with exit flow coefficient for Radiver impeller. Compared with CFD results, Oh's model underpredicts the exit aerodynamic blockage, because it doesn't consider the interaction of the shock wave with the tip leakage flow. The improved model over-predicts the exit aerodynamic blockage in the high flow coefficient region, and under-predicts near the stall condition. A maximum error of about $9.67 \%$ occurs near the stall condition. Compare with Oh's model, the improved model shows better accuracy with the RMSE of about $6.15 \%$.

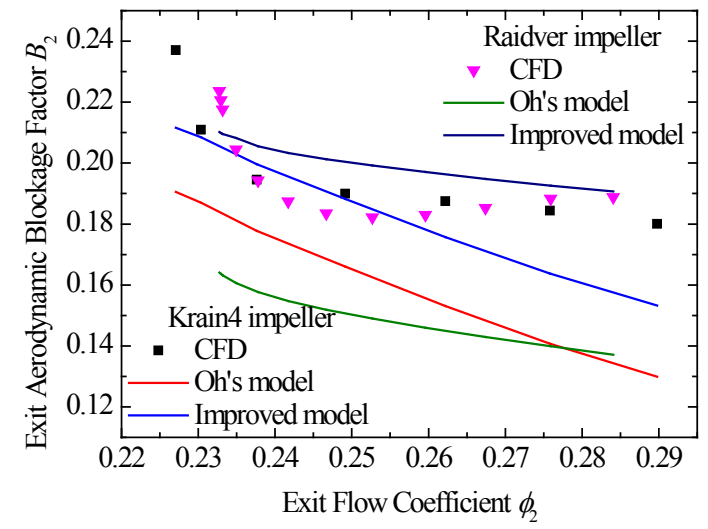

Figure 20 Comparison between the CFD and model predicting exit aerodynamic blockage with exit flow coefficient

Figure 21 shows the exit aerodynamic blockage predicted by models with rotational speed for Radiver impeller. Compared with CFD results, the improved model over-predicts the exit aerodynamic blockage in the low rotational speed region, and under-predicts in the high rotational speed region. A maximum error of about $17.30 \%$ occurs at the $1.1 n_{\mathrm{d}}$ speed line. Compare with Oh's model, the improved model shows better accuracy with the RMSE of about $9.79 \%$.

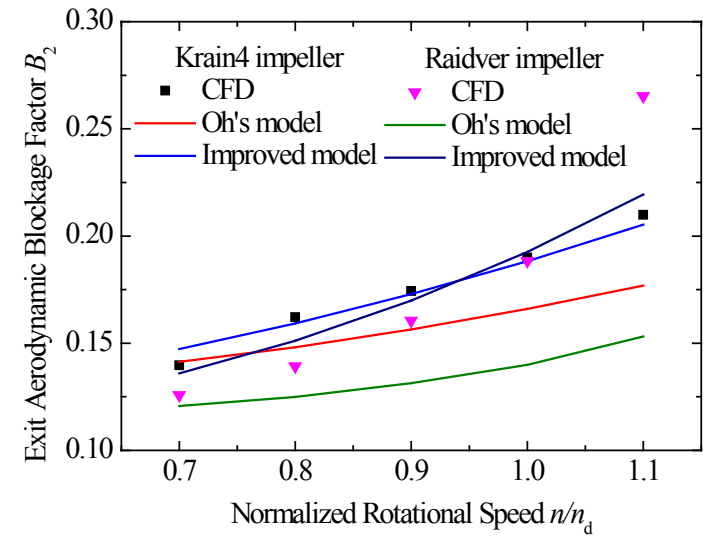

\section{Figure 21 Comparison between the CFD and model predicting exit aerodynamic blockage with rotational speed $\left(n=n_{\mathrm{d}}, \phi_{2}=0.249\right)$}

Figure 22 shows the exit aerodynamic blockage predicted by models with tip clearance size for Radiver impeller. Compared with CFD results, the improved model over-predicts the exit aerodynamic blockage with large tip clearance size, and under-predicts with small tip clearance size. A maximum error of about $5.18 \%$ occurs at the largest tip clearance size. Compare with Oh's model, the improved model shows better accuracy with the RMSE of about $3.60 \%$. 


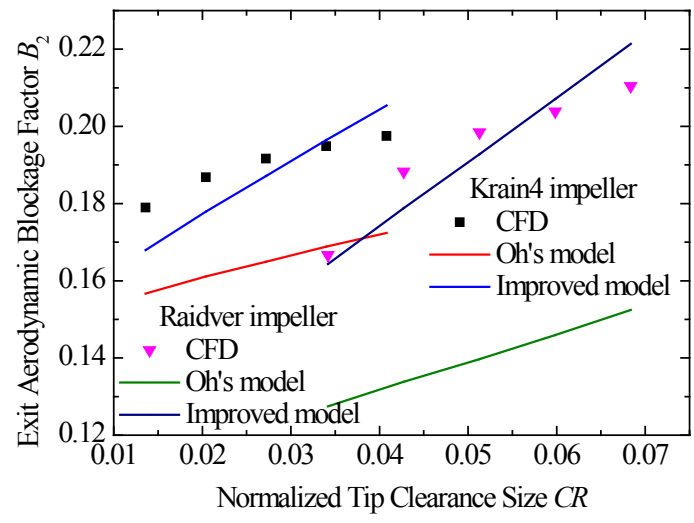

Figure 22 Comparison between the CFD and model predicting exit aerodynamic blockage with tip clearance size ( $\left.\phi_{2}=0.249\right)$

\section{CONCLUSIONS}

This study investigates the effect of the exit flow coefficient, rotational speed and tip clearance size on the exit aerodynamic blockage. An improved one-dimensional model is proposed to predict the exit aerodynamic blockage factor for transonic centrifugal impellers. Owing to its speed, and ease of use, this model is eminently suitable for preliminary design calculations.

The main conclusions of this paper are as follows:

- The decrease of exit flow coefficient, the enhancement of rotational speed, and the increase of tip clearance size can increase exit aerodynamic blockage.

- The inlet blockage had a great effect on the exit blockage. The decrease of exit flow coefficient and the decrease of rotational speed can increase inlet aerodynamic blockage. The intensity of the shock wave dominates the inlet blockage, compared with tip clearance.

- The proposed exit aerodynamic blockage prediction model considers the interaction of the shock wave with the tip leakage flow, based on Oh's model. Compared with conventional model, the current model has better accuracy, with an average relative error below $9.79 \%$.

\section{ACKNOWLEDGMENTS}

This research was supported by National Natural Science Foundation of China (No. 51676182).

\section{NOMENCLATURE}

\section{Notations}

A Passage area

$B \quad$ Aerodynamic blockage

$V \quad$ Absolute flow velocity

$W \quad$ Relative flow velocity

$Z \quad$ Blade number

$b \quad$ Blade width

c Tip clearance size

d Diameter

$m \quad$ Mass flow rate

$\begin{array}{ll}n & \text { Rotational speed } \\ n u m & \text { Amount of samples at the all operation conditions } \\ r & \text { Radius } \\ t & \text { Blade thickness } \\ A R & \text { Geometric area ratio } \\ D R & \text { Diffusion ratio } \\ C R & \text { Tip clearance ratio } \\ \text { LE } & \text { Leading edge } \\ M R & \text { Inlet tip relative Mach number ratio } \\ \text { PS } & \text { Pressure surface } \\ \text { RMSE } & \text { Root-mean-square error } \\ \text { SS } & \text { Suction surface } \\ \text { TE } & \text { Trailing edge } \\ \text { TLV } & \text { Tip leakage vortex } \\ C_{\mathrm{ps}} & \text { Static pressure coefficient } \\ M_{\mathrm{w} 1 \mathrm{t}, \mathrm{cr}} & \text { Critical inlet tip relative Mach number } \\ r_{\mathrm{hyd}} & \text { Passage hydraulic radius } \\ \beta & \text { Relative angle from meridional direction } \\ \rho & \text { Density } \\ \phi_{2} & \text { Exit flow coefficient } \\ \Delta C_{p s} & \text { Static pressure coefficient difference between the } \\ & \text { blade surfaces }\end{array}$

\section{Subscripts}

$\begin{array}{ll}0 & \text { Stagnation parameter } \\ 1 & \text { Impeller inlet } \\ 2 & \text { Impeller exit } \\ 3 & \text { Vaneless diffuser exit } \\ b & \text { Blade parameter } \\ d & \text { Design parameter } \\ \text { eff } & \text { Effective parameter } \\ m & \text { Meridional component; RMS } \\ t & \text { Tip parameter } \\ t h & \text { Throat parameter } \\ M & \text { Predicted value by model } \\ N & \text { Predicted value by CFD }\end{array}$

Superscripts

- Mass-averaged

\section{REFERENCES}

CASEY, M. \& ROBINSON, C. 2010. A new streamline curvature throughflow method for radial turbomachinery. Journal of Turbomachinery, 132, 031021.

CHIMA, R. V. 1998. Calculation of Tip Clearance Effects in a Transonic Compressor Rotor. Journal of Turbomachinery, 120, 131-140.

DU, J., LIN, F., CHEN, J., NIE, C. \& BIELA, C. 2013. Flow Structures in the Tip Region for a Transonic Compressor Rotor. Journal of Turbomachinery, 135, 031012-031012-11.

HAH, C. \& KRAIN, H. 1990. Secondary Flows and Vortex Motion in a High-Efficiency Backswept Impeller at Design and Off-Design Conditions. Journal of Turbomachinery, ASME Paper, 1-10.

IBARAKI, S., FURUKAWA, M., IWAKIRI, K. \& TAKAHASHI, K. 2007. Vortical Flow Structure and Loss Generation Process in a Transonic Centrifugal Compressor Impeller. 1089-1098. 
KAI, U. Z., GALLUS, H. E. \& NIEHUIS, R. 2002. A Study on Impeller-Diffuser Interaction-Part II: Detailed Flow Analysis. Journal of Turbomachinery, 125, 183-192.

KAI, U. Z., GALLUS, H. E. \& NIEHUIS, R. 2003. A Study on Impeller-Diffuser Interaction-Part I: Influence on the Performance. Journal of Turbomachinery, 125, 545-556.

KANEKO, M. \& TSUJITA, H. 2015. Mechanism of Blockage Generation in Transonic Centrifugal Compressor at Design and Off-Design Conditions. V02CT42A025.

KRAIN, H. 1984. A CAD-Method for Centrifugal Compressor Impellers. Journal of Engineering for Gas Turbines \& Power, 106, 1-7.

KRAIN, H. 1988. Swirling impeller flow. Journal of Turbomachinery, Vol. 110, 122-128.

OH, J. \& OH, K. 2000. Numerical Modeling of Some Parameters for Performance Prediction of Centrifugal Impellers. V001T03A032.

PAMPREEN, R. C. 1981. A Blockage Model for Centrifugal Compressor Impellers. Journal of Engineering for Power, 103, 698-707.

RODGERS, C. 1978. A Diffusion Factor Correlation for Centrifugal Impeller Stalling. Journal of Engineering for Power, 100, 592-601.

SENOO, Y., MARUYAMA, S., KOIZUMI, T. \& NAKASE, Y. 1974. Viscous Effects on Slip Factor of Centrifugal Blowers. Journal of Engineering for Power, 96, 59-65.

YAMADA, K., FUNAZAKI, K. \& FURUKAWA, M. 2007. The Behavior of Tip Clearance Flow at Near-Stall Condition in a Transonic Axial Compressor Rotor. 295-306.

YI, W., CHEN, Z.-M. \& JI, L. 2014. Numerical Studies on Application of Blended Blade and Endwall Technique on Transonic Centrifugal Compressor. V02DT42A033. 